\title{
LASER BEAM CHARACTERIZATION BY MEANS OF DIFFRACTIVE OPTICAL CORRELATION FILTERS
}

\author{
V.S. Pavelyev, V.A. Soifer, M. Duparre*, B. Luedge* \\ Image Processing Systems Institute, Russian Academy of Sciences, Samara, Russia \\ *Institute for Applied Optics, Univ. of Jena, D-07743 Jena, Germany
}

Analyzing of amplitude-phase characteristics of laser beam is topical in experimental physics and in a great number of laser applications, such as, for example, laser material treatment. The task of analyzing the amplitude-phase beam structure may be treated as that of analyzing the modal composition, if this is thought of as both analyzing individual modal powers and intermode phase shifts. In this paper the problem is tackled using a special diffractive optical element (DOE), called MODAN, matched to a group of laser radiation modes and their special combinations. The experimental results reported indicate that such an approach shows promise.

Key words: laser beam, Gaussian modes, intermode power distribution, intermode phase shifts.

\section{Introduction}

The analysis of amplitude-phase characteristics of the laser beam is a topic in experimental physics and in a great number of laser applications, such as laser material treatment $[1,2,3]$. In this paper the problem is tackled using a special diffractive optical element (DOE) MODAN - matched to a group of transversal laser modes. Laser radiation modes are beams whose crosssection complex amplitude distributions are described by eigenfunctions of the operator of light propagation [3]. Most familiar are the Bessel or free space modes [4], and the Gaussian (Gauss-Hermite and GaussLaguerre) modes [3,5]. A fundamental property of the Gaussian modes is their ability to retain structure and mutual orthogonality while propagating in free space or lens-like medium [3,5]. The Gaussian modes describe the modes of an open passive resonator with spherical mirrors, and the modes of a gradient-index fiber with parabolic profile $[3,5]$. Coherent light modes may be interpreted as "generalized spectral components" since they propagate in their medium with modal eigenvalue and individual transverse amplitude-phase structure, acquiring only a phase shift [3] proportional to the optical path passed. Having in mind the above mode concept, we can represent any given amplitude-phase light distribution $W(\mathbf{u})$ as a superposition of a number of modes $\psi_{\mathbf{n}}(\mathbf{u})$ with definite complex-valued coefficients $C_{\mathbf{n}}$ [3]:

$$
W(\mathbf{u})=\sum_{\mathbf{n}=\mathbf{0}}^{\infty} C_{\mathbf{n}} \psi_{\mathbf{n}}(\mathbf{u}),
$$

where $\mathbf{n}=(n, m), \mathbf{u}=(u, v)$. Thus, analyzing the amplitude-phase structure of the beam reduces to analyzing the modal composition understood as measurement of both individual modal powers, $\left|C_{n}\right|^{2}$, and intermodal phase shifts, $\Delta \varphi_{\mathrm{n}}=\arg C_{\mathrm{n}}-\arg C_{\mathrm{n}-1}$. Then, based on the measurements made for $N_{\bmod }$ modes we can reconstruct the initial amplitude-phase distribution taking any arbitrary constant value as phase $\varphi_{0}=\arg C_{0}$ :

$$
\begin{aligned}
& W(\mathbf{u}) \cong\left|C_{0}\right| \exp \left(\mathrm{i} \cdot \varphi_{0}\right) \psi_{0}(\mathbf{u})+ \\
& +\sum_{\mathrm{n}^{\prime}=1}^{N}\left|C_{\mathrm{n}^{\prime}}\right| \psi_{\mathrm{n}^{\prime}}(\mathbf{u}) \exp \left[\mathrm{i}\left(\Delta \varphi_{\mathrm{n}^{\prime}}+\arg C_{\mathrm{n}^{\prime}-1}\right)\right]^{-1}
\end{aligned}
$$

$$
\arg C_{\mathrm{n}^{\prime}}=\arg C_{\mathrm{n}^{\prime}-1}+\Delta \varphi_{\mathrm{n}^{\prime}}
$$

The accuracy of the estimate in Eq. (2) of the series in Eq. (1) have been discussed in [3]. We just note that in most practical tasks it is the maximal value of the mode $\mathbf{N}_{\text {cut }}$ with non-zero coefficient $C_{\mathbf{N}_{\text {cut }}} \neq 0$ in Eq. (1) which is known, so in practical applications $N_{\text {mod }}=f\left(\mathbf{N}_{\text {cut }}\right)$. Using the measured individual mode powers and intermodal phase shifts for modes ranging from fundamental one to that cut-off allows the amplitude-phase distribution in the laser beam crosssection to be reconstructed. Numerical methods discussed in $[6,7]$ make it possible to reconstruct the power distribution between the modes, while the methods in Refs. [8,9] provide the direct reconstruction of the amplitude-phase distribution in the beam cross-section by numerical processing of the results of measuring the beam intensity in various planes. Note, however, that such an approach requires a high-precision measurement of intensity, analog-to-digital transformation and further numerical processing of intensity distributions or interferograms. Besides, the iterative phase reconstruction $[8,9]$ from the results of intensity measurements is fundamentally unable to guarantee the convergence of the iterative procedure. In $[3,10]$ it has been shown that the use of fundamental properties of laser light modes makes possible the synthesis of diffractive optical elements - MODANS - for analysis of modal composition. Rapid progress in technologies for DOE fabrication, including those for high-power lasers [11], open wide prospects to the use of such elements in the studies of the laser beam cross-section structure.

\section{Basic formalism: DOE design for analysis of the beam modal spectrum}

In [3] they report on the designing of phase modans intended for the analysis of the coherent beam transversal modal spectrum. To change from the complex-valued transmission function (in the general case) to the purely-phase function both analytical coding methods [3] (with a carrier introduced into the element's phase) and iterative coding methods [9] have been developed. 


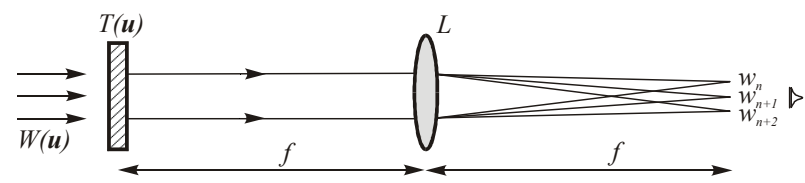

Fig. 1. Formulation of the problem: analysis of the beam modal composition

To this end, we need to seek for the DOE complex transmission function as a superposition of the complex transmission functions of "virtual" subapertures with corresponding weight coefficients.

"Virtual" apertures correspond to optical filters matched to the modes (or their combinations) and intended for deriving information about the modal powers, $\left|C_{\mathrm{n}^{\prime}}\right|^{2}$, and/or intermodal phase shifts $\Delta \varphi_{\mathrm{n}^{\prime}}$.

Let us consider the calculation of the complex transmission function for such an element in more detail. In [3] they have shown that if a beam of cross-section complex-valued distribution $W(\mathbf{u})$ interacts with a filter of complex transmission function $\psi^{*} \mathbf{n}(\mathbf{u})$ (where $\psi_{\mathbf{n}}(\mathbf{u})$ is the mode numbered $\mathbf{n}$, and $*$ denotes complex conjugation) at the Fourier-stage input, the resulting complex distribution at the center of the Fourier-stage output plane will be given by

$$
w(\mathbf{0})=\iint_{D} W(\mathbf{u}) \psi^{*}{ }_{\mathbf{n}}(\mathbf{u}) \mathrm{d}^{2} \mathbf{u} \cong C_{\mathbf{n}},
$$

where $C_{\mathbf{n}}$ is the modal coefficient of the $\mathbf{n}$-th mode in the decomposition in Eq. (1) and $D$ is the element aperture.

Measuring with a point-wise pick-off the intensity $\left|C_{\mathbf{n}}\right|^{2}$ at the output plane center allows one to derive information about the energy contribution of the mode number $\mathbf{n}$ into the beam $W(\mathbf{u})$. To measure the $N$ mod modal powers

simultaneously, in [3] they consider an element with the complex transmission function in the form

$$
T(\mathbf{u})=\sum_{\mathrm{n}^{\prime}=1}^{N_{\text {mod }}} \zeta_{\mathrm{n}^{\prime}} \psi^{*}{ }_{\mathrm{n}^{\prime}}(\mathbf{u}) \exp \left(\mathrm{i} v_{\mathrm{n}^{\prime}} \mathbf{u}\right) .
$$

The exponential additions $\exp \left(\mathrm{i} v_{\mathrm{n}^{\prime}} \mathbf{u}\right)$ are introduced to spatially separate channels corresponding to different modes in the element's plane, and the weight coefficients $\zeta_{\mathrm{n}^{\prime}} \geq 0$ are introduced to produce the desired energy redistribution between the subapertures (if needed). The interaction of the beam with a filter of complex transmission function in Eq. (4) at the input of a Fourier-stage of focal length $f$ will result in the emergence of complex distributions $w\left(\lambda f v_{\mathrm{n}^{\prime}}\right) \cong \zeta_{\mathrm{n}^{\prime}} C_{\mathrm{n}^{\prime}}$ in the output plane (Fig. 1). In [3], to measure the intermode phase shifts $\Delta \varphi_{n^{\prime}}=\arg C_{n^{\prime}}-\operatorname{argC} C_{n^{\prime}-1}$ by modal optical correlation filters they introduce into the modan phase of extra virtual subapertures with the transmission functions given by

$$
\xi_{\mathrm{k}}\left[\psi^{*}{ }^{*}{ }^{\prime}-1(\mathbf{u})+\psi^{*}{ }^{*}(\mathbf{u})\right] \exp \left(\mathrm{i} v_{\mathrm{k}} \mathbf{u}\right),
$$

$$
\widetilde{\zeta}_{\mathrm{s}}\left|\psi^{*}{ }^{\mathrm{n}^{\prime}-1}(\mathbf{u}) \exp (\mathbf{i} \Delta \widetilde{\varphi})+\psi^{*}{ }^{\prime}{ }^{\prime}(\mathbf{u}) \cdot\right| \exp \left(\mathrm{i} \nu_{\mathrm{s}} \mathbf{u}\right)
$$

where $\Delta \widetilde{\varphi}$ is a certain phase shift introduced. It can be demonstrated [3] that the intensity values $\left|w_{\mathrm{k}}\right|^{2}=\left|w\left(\lambda f v_{\mathrm{k}}\right)\right|^{2}$ and $\left|w_{\mathrm{s}}\right|^{2}=\left|w\left(\lambda f v_{\mathrm{s}}\right)\right|^{2}$ are defined as follows (lets assume $\xi=1, \widetilde{\zeta}=1$ ):

$$
\begin{aligned}
& \left|\mathrm{w}_{\mathrm{k}}\right|^{2} \cong\left|\mathrm{w}_{\mathrm{n}^{\prime}-1}\right|^{2}+\left|\mathrm{w}_{\mathrm{n}^{\prime}}\right|^{2}+ \\
& +2 \cdot\left|\mathrm{w}_{\mathrm{n}^{\prime}-1}\right| \cdot\left|\mathrm{w}_{\mathrm{n}^{\prime}}\right| \cos \left(\Delta \varphi_{\mathrm{n}^{\prime}}\right) \\
& \left|\mathrm{w}_{\mathrm{s}}\right|^{2} \cong\left|\mathrm{w}_{\mathrm{n}^{\prime}-1}\right|^{2}+\left|\mathrm{w}_{\mathrm{n}^{\prime}}\right|^{2}+2 \cdot\left|\mathrm{w}_{\mathrm{n}^{\prime}-1}\right| \cdot \\
& \cdot\left|\mathrm{w}_{\mathrm{n}^{\prime}}\right|\left[\cos \left(\Delta \varphi_{\mathrm{n}^{\prime}}\right) \cos \Delta \widetilde{\varphi}-\sin \left(\Delta \varphi_{\mathrm{n}^{\prime}}\right) \sin \Delta \widetilde{\varphi}\right]
\end{aligned}
$$

Then, as it has been shown in [3], the values of the intermode phase shifts $\Delta \varphi_{\mathrm{n}^{\prime}}$ can be derived from Eqs. (7) and (8). Knowing the maximal admissible mode number in the beam under analysis we can write the relation for the complex transmission function of a filter intended for the analysis of the beam amplitude-phase composition:

$$
\begin{aligned}
& T(\mathbf{u})=\sum_{\mathrm{n}^{\prime}=1}^{N_{\text {mod }}} \zeta_{\mathrm{n}^{\prime}} \psi^{*}{ }_{\mathrm{n}^{\prime}}(\mathbf{u}) \exp \left(\mathrm{i} v_{\mathrm{n}^{\prime}} \mathbf{u}\right)+ \\
& +\sum_{\mathrm{k}=1}^{N_{\text {mod }}-1} \xi_{\mathrm{k}}\left[\psi^{*}{ }_{\mathrm{k}}(\mathbf{u})+\psi^{*}{ }_{\mathrm{k}+1}(\mathbf{u})\right] \exp \left(\mathrm{i} v_{\mathrm{k}} \mathbf{u}\right)+ \\
& +\sum_{\mathrm{s}=1}^{N_{\text {mod }}{ }^{-1}} \widetilde{\zeta}_{\mathrm{s}}\left[\psi^{*}{ }_{\mathrm{s}}(\mathbf{u}) \exp \left(\mathrm{i} \Delta \widetilde{\varphi}_{\mathrm{s}}\right)+\psi^{*}{ }_{\mathrm{s}+1}(\mathbf{u})\right] \times \\
& \times \exp \left(\mathrm{i} v_{\mathrm{s}} \mathbf{u}\right)
\end{aligned}
$$

Taking into account that certain mode(s) $\psi_{\mathrm{k}}(\mathbf{u})$ can have zero-valued coefficient $C_{\mathrm{k}}=0$, Eq.(9) should be changed to

$$
\begin{aligned}
& T(\mathbf{u})=\sum_{\mathrm{n}^{\prime}=1}^{N_{\text {mod }}} \zeta_{\mathrm{n}^{\prime}} \psi^{*}{ }_{\mathrm{n}^{\prime}}(\mathbf{u}) \exp \left(\mathrm{i} v_{\mathrm{n}^{\prime}} \mathbf{u}\right)+ \\
& +\sum_{\mathrm{k}=0, \mathrm{k} \neq \mathrm{r}-1}^{N_{\text {mod }}-1} \xi_{\mathrm{k}}\left[\psi^{*}{ }_{\mathrm{r}}(\mathbf{u})+\psi^{*}{ }_{\mathrm{k}+1}(\mathbf{u})\right] \exp \left(\mathrm{i} v_{\mathrm{k}} \mathbf{u}\right)+ \\
& +\sum_{\mathrm{s}=0, \mathrm{~s} \neq \mathrm{r}-1}^{N_{\text {mod }}{ }^{-1}} \widetilde{\zeta}_{\mathrm{s}}\left[\psi^{*}{ }_{\mathrm{r}}(\mathbf{u}) \exp \left(\mathrm{i} \Delta \widetilde{\varphi}_{s}\right)+\psi^{*}{ }_{\mathrm{s}+1}(\mathbf{u}) \cdot\right] \times \\
& \times \exp \left(\mathrm{i} v_{\mathrm{s}} \mathbf{u}\right)
\end{aligned}
$$

where $r$ is the number of the "reference" mode, which has always nonzero valued coefficient $C_{\mathrm{r}} \neq 0$ (for the most of real laser resonators this can be the fundamental mode $\mathbf{n}=(0,0))$. Now, based on the measurements made taken for $N_{\text {mod }}$ modes we can estimate the initial amplitude-phase distribution except a constant phase shift of $\arg C_{\mathrm{r}}=\varphi_{\mathrm{r}}$ :

$$
\begin{aligned}
& W(\mathbf{u}) \cong\left|C_{\mathrm{r}}\right| \exp \left(\mathrm{i} \cdot \varphi_{\mathrm{r}}\right) \psi_{\mathrm{r}}(\mathbf{u})+ \\
& +\sum_{\mathrm{n}^{\prime}=1, \mathrm{n}^{\prime} \neq \mathrm{r}}^{N_{\text {mod }}} C_{\mathrm{n}^{\prime}} \mid \psi_{\mathrm{n}^{\prime}}(\mathbf{u}) \exp \left[\mathrm{i}\left(\Delta \phi_{\mathrm{n}^{\prime}}+\varphi_{\mathrm{r}}\right)\right], \\
& \Delta \oint_{\mathrm{n}^{\prime}}=\arg C_{\mathrm{n}^{\prime}}-\varphi_{\mathrm{r}} .
\end{aligned}
$$




\section{Design and manufacturing of a diffractive optical element for mode power distribution measurement}

The derived complex transmission function in Eq. (4) (which is certainly not phase-only in general case) can be reduced to the purely phase function using wellknown encoding techniques [3] based on the introduction of a carrier into the phase function, for example, by generalization of well-known Kirk-Jones method [12] described in [3]. Thus, the modan for measurement of modal power distribution for 10 Gaussian-Laguerre modes was calculated by Eq. (4) and manufactured and investigated in the frame of present paper. The analyzing MODAN used for the investigations presented in this paper was designed to detect 10 GaussLaguerre(GH)-modes which are below the chosen cutoff condition $2 n+|m| \leq 3:(n, m)=(0,0),(1,0),(0,1)$, $(1,1),(0,2),(0,3),(0,-1),(1,-1),(0,-2),(0,-3)$. In accordance with our experimental conditions, the design value of the beam size parameter for these ten modes was selected as $\sigma=0.8 \mathrm{~mm}$. The element was calculated for a wavelength of $\lambda=632.8 \mathrm{~nm}$, and should work together with a Fourier lens of $f=452 \mathrm{~mm}$. The corresponding ,correlation points“ for each mode, respectively, should develop in the focal plane of this lens in a distance of $1.5 \mathrm{~mm}$ from each other (see Fig. 3). The element was coded as a grating with 55.5 lines $/ \mathrm{mm}$, with a rectangular shaped carrier function ,slowly“ modulated across the aperture (,synthetic coefficients' method"). The grating was calculated with a resolution of 2048 x 2048 pixels, with a pixel size of $3.0 \mu \mathrm{m} \times 3.0$ $\mu \mathrm{m}$. Figure 2 depicts the calculated phase distribution at the aperture of modan. Fig. 3 depicts the amplitude distribution in the used (zeroth) diffraction order of the element under the suggestion that it was illuminated by a pure $(0,0)$ gaussian mode with $\sigma=0.8 \mathrm{~mm}$.

The calculated phase would have to be transferred into a corresponding surface depth, taking into consideration the intended transmission mode of the element, and the refractive index of the resist. In a next step, this two-dimensional ,continuous“ surface pixel map should be copied out into a corresponding electron dose map, which in its turn could have been written subsequently by the e-beam lithograph. However, thermal load resulting from such a one-shot-per-pixel procedure negatively influences final resist- and surface quality. As a consequence, the „continuous“ surface pixel map was approximated by a staircase structure with 16 permitted levels in depth (=15 steps of equal height in the staircase). Each of these 15 steps had to be generated by an individual „etching“ process. Therefore, 15 single binary masks had to be generated (as data fields) by software, starting from the „continuous“ surface pixel map. Manufacturing started from a substrate from quartz glass, covered with a thin film of PMMA (thickness nearly $2.5 \mu \mathrm{m}$ ), into which the spatially varying dose of electrons was implanted. The 15 dose levels each corresponding to one of the future new surface levels were realized by 15 times application of a binary electron beam writing process, using a commercial ZBA $23 \mathrm{H}$ system. After finishing the development procedure of the PMMA film, the profile depth over all 15 steps should be $1200 \mathrm{~nm}$.

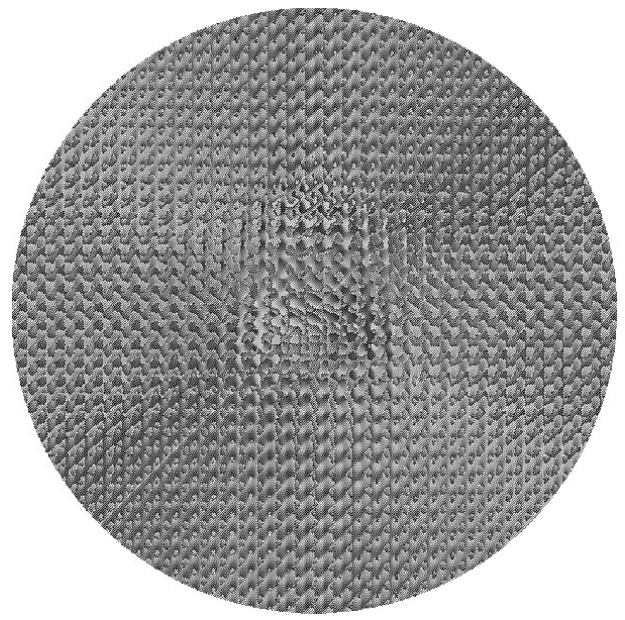

Fig.2. Calculated phase distribution at the aperture of modan

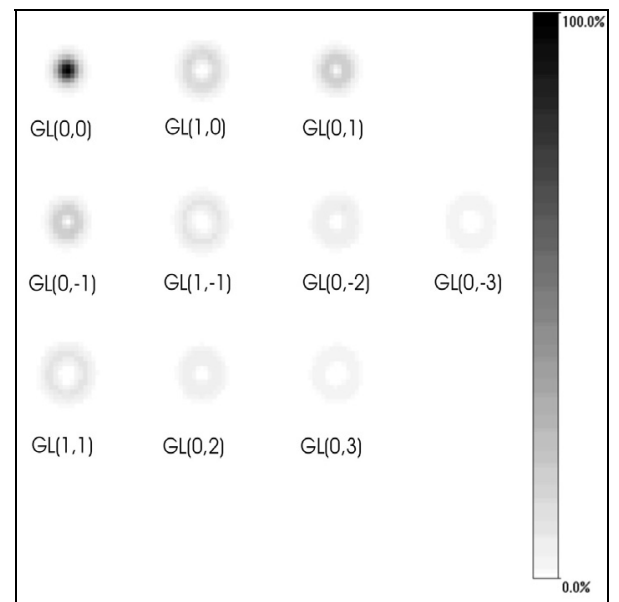

Fig. 3. Result of simulation of detection of the $(0,0)$ mode by the designed DOE

\section{Optical set-up}

The manufactured analyzing MODAN had to demonstrate its performance in a series of optical experiments. For this purpose, the set-up schematically shown in Fig. 4 has been used. The 10-channel modan $(\sigma=0.8 \mathrm{~mm})$ has been illuminated by gaussian beam with $\sigma=0.525 \mathrm{~mm}$ (see Fig. 5,6). The decomposition of gaussian distribution with $\sigma=0.525 \mathrm{~mm}$ by using basis of Gaussian-Laguerre modes with $\sigma=0.8 \mathrm{~mm}$ giving us non-zero values of $(0,0)$ mode coefficient and $(1,0)$ mode coefficient (nearly $25 \%$ of $(0,0)$ coefficient). Another modes with nonzero coefficients in this decomposition are of order higher then cutoff number was used.

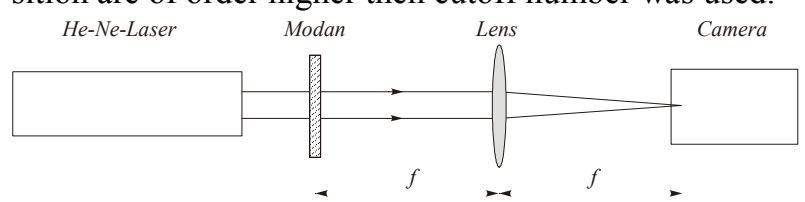

Fig.4. Optical set-up for modan investigation 


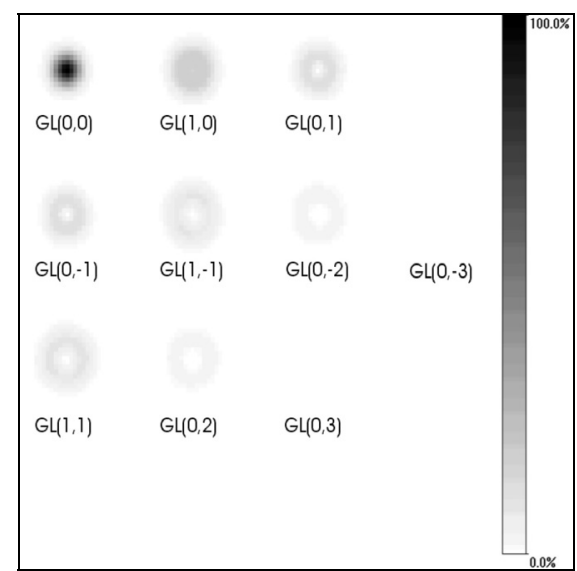

Fig. 5. Result of simulation of illumination of modan by $(0,0)$-mode with $\sigma=0.525 \mathrm{~mm}$

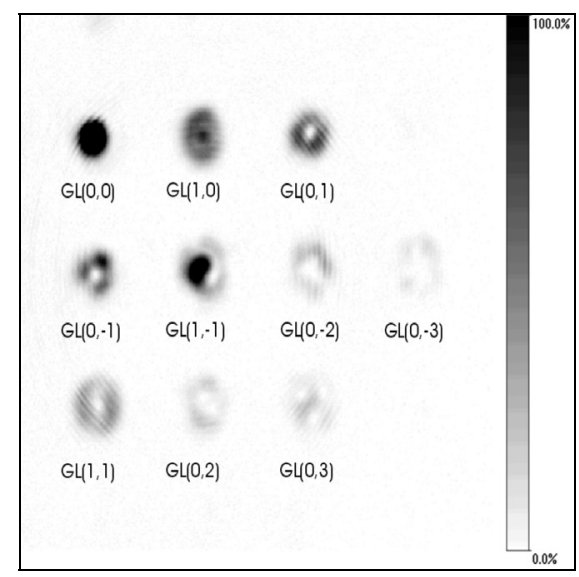

Fig. 6. Result of measurement of intensity in the output plane of modan illuminated by $(0,0)$-mode with $\sigma=0.525 \mathrm{~mm}$

Note good agreement between results of computational and natural experiments. Note also parasitical intensity peak in the central diffractive order (at the place of $(1,-1)$ mode correlation peak) which is connected with technological problem - calculated surface profile was not perfectly achieved.

\section{Conclusion}

Designing a diffractive optical element intended for the analysis of the amplitude-phase composition of a laser beam has been reported in this paper. The results of computer simulation reported confirm the potentialities of the developed approach.

\section{Acknowledgements}

The authors would like to thank B. Kley from IAP for assistance in manufacturing the $3 \mathrm{~d}$ resist profile.

\section{References}

1. Ward B.A., "In-process sampling and analysis of the far-field of a $21 \mathrm{~kW} \mathrm{CO}$ laser beam" in Laser Beam Characterization, P. M. Mejias, H. Weber, R. Martinez-Herrero and A. Gonzales-Urena, editors, (SEDO, Madrid, 1993), 53-64.

2. Teppo E. A., "Diagnostic tools for laser beam characterization" in Laser Beam Characterization, P. M. Mejias, H. Weber, R. Martinez-Herrero and A. Gonzales-Urena, editors, (SEDO, Madrid, 1993), 23-30.

3. Soifer V.A., Golub M.A., Laser Beam Mode Selection by Computer Generated Holograms .- 1994.CRC Press. 215 p.

4. Durnin J., Miceli J.J., Eberly Jr. And J.H., ’Diffraction-Free Beams", Physical Review Letters, 58 (15), 1499-1501.

5. Yariv A., Optical electronics, Holt, Rinehart, and Winston, New York, 1985.

6. Cutolo A., Pierri T., Zeni L., "Measurement of the mode content of a laser beam" in Laser Beam Characterization, P. M. Mejias, H. Weber, R. MartinezHerrero and A. Gonzales-Urena, editors, (SEDO, Madrid, 1993), 263-273.

7. Du K.-M., Herziger G., Loosen P., Ruhl F., "Measurement of the mode coherence coefficients", Optical and Quantum Electronics, 24, S1119- S1127, (1992).

8. Stark H., Image Recovery: Theory and Application, Academic Press Inc., 1987.

9. Soifer V.A., Kotlyar V.V., Doscolovich L.L., Iterative Methods for Diffractive Optical Elements Computation, Taylor\&Francis Ltd., 1997

10. Golub M.A., Prokhorov A.M., Sisakian I.N., Soifer V.A., "Synthesis of spatial filters for investigation of transversal modal content of coherent radiation", Quantum Electronics, 9, N 9, 1866-1868, (1982).

11. Kononenko V.V., Konov V. I., Pimenov S. M., Prokhorov A. M., Pavelyev V. S., Soifer V. A., „CVD diamond transmissive diffractive optics for $\mathrm{CO}_{2}$ lasers", New Diamond and Frontier Carbon Technology, 10, N 2, (2000) (accepted for publication).

12. Kirk J.P., Jones A.L., "Phase-only complex valued spatial filter", JOSA, 61, N 8 , 1023-1028, (1971).

13. Duparre M., Pavelyev V.S., Ludge B., Soifer V.A., Kowarschik R., "Laser beam characterization by means of optical correlation filters", Proceedings of EOS Topical Meeting in Diffractive Optics DO99, Jena, Germany 23-25 August 1999, 284-285. 